\title{
Coherent Control of Quantum Chaotic Diffusion
}

\author{
Jiangbin Gong and Paul Brumer \\ Chemical Physics Theory Group, University of Toronto, Toronto, Canada M5S $3 H 6$
}

(Received 30 May 2000)

\begin{abstract}
Extensive coherent control over quantum chaotic diffusion using the kicked rotor model is demonstrated and its origin in deviations from random matrix theory is identified. Further, the extent of control in the presence of external decoherence is established. The results are relevant to both areas of quantum chaos and coherent control.
\end{abstract}

DOI: 10.1103/PhysRevLett.86.1741

The kicked rotor and its classical limit, the standard map, have long served as paradigms for classical and quantum chaos [1]. The classical dynamics shows characteristic diffusive energy growth whereas the quantum dynamics shows similar chaotic short time behavior, followed by the suppression of diffusion at longer times. In this Letter, we demonstrate that the quantum features of the chaotic kicked rotor allow for extensive coherent control [2] over quantum chaotic diffusion, even in the presence of modest decoherence. In particular, we show that quantum relaxation dynamics in the kicked rotor model is sensitive to the coherence characteristics of the initial state, and that altering these characteristics allows for control over the energy diffusion. The extent of the controlled behavior is vast, from strong suppression to strong enhancement of diffusion.

Consider the kicked rotor whose Hamiltonian is given by

$$
H(\hat{L}, \theta, t)=\frac{\hat{L}^{2}}{2 I}+\lambda \cos (\theta) \sum_{n} \delta(t / T-n),
$$

where $\hat{L}$ is the angular momentum operator, $\theta$ is the conjugate angle, $I$ is the moment of inertia, $\lambda$ is the strength of the "kicking field," and $T$ is the time interval between kicks. The quantum time evolution operator $\hat{F}$ for times $(N-1 / 2) T$ to $(N+1 / 2) T$ is [1]

$$
\hat{F}=\exp \left[i \frac{\tau}{4} \frac{\partial^{2}}{\partial \theta^{2}}\right] \exp [-i k \cos (\theta)] \exp \left[i \frac{\tau}{4} \frac{\partial^{2}}{\partial \theta^{2}}\right]
$$

with dimensionless parameters $\tau=\hbar T / I$ and $k=\lambda T / \hbar$. The classical limit [1] of this quantum map is given by the standard map, which, when expressed in terms of dimensionless variables $\theta$ and the scaled $c$-number angular momentum $\tilde{L}=L \tau / \hbar$, takes the following form:

$$
\begin{aligned}
& \theta_{N}=\theta_{N-1}+\left(\tilde{L}_{N}+\tilde{L}_{N-1}\right) / 2, \\
& \tilde{L}_{N}=\tilde{L}_{N-1}+\kappa \sin \left(\theta_{N-1}+\tilde{L}_{N-1} / 2\right),
\end{aligned}
$$

where $\kappa=k \tau$, and $\left(\tilde{L}_{N}, \theta_{N}\right)$ represents the phase space location of a classical trajectory after $N$ kicks. The system is chaotic for $\kappa>\kappa_{c r}=0.9716 \ldots$ The resultant diffusion constant can be defined as the absorption rate of the average scaled rotational energy $\tilde{E} \equiv\left\langle\tilde{L}^{2}\right\rangle / 2$. Com-
PACS numbers: $05.45 . \mathrm{Gg}$, 05.45.Mt, 05.60.Gg, 32.80.Qk

paring classical and quantum dynamics for typical initial classical states shows that quantum dynamics displays significant suppression of the classical chaotic diffusion, i.e., the external field can only excite a finite number of unperturbed energy levels [3].

The fact that the rotor is a Hamiltonian system and the kick is coherent implies that the system maintains its quantum phase throughout the evolution. If this is the case then the system should be controllable via coherent control [2], i.e., by using quantum interference phenomena to affect the dynamics. To demonstrate this, and to examine the extent of possible control, we consider the dynamics of states which are initially composed of superpositions of two arbitrary angular momentum eigenstates, $|m\rangle=\exp (\operatorname{im} \theta) / \sqrt{2 \pi}$ and $|n\rangle=\exp (\operatorname{in} \theta) / \sqrt{2 \pi}$. Each of these eigenstates is classically allowed, with a corresponding classical distribution function given by $\rho_{m}^{c}(\theta, \tilde{L})=\delta_{\tilde{L} / \tau, m} / 2 \pi$ and $\rho_{n}^{c}(\theta, \tilde{L})=\delta_{\tilde{L} / \tau, n} / 2 \pi$, respectively [4].

To show that changing the coherent characteristics of the initial state significantly alters the subsequent dynamics, we consider the dynamics of states given initially by the superposition $|\psi\rangle=\cos (\alpha)|m\rangle+\sin (\alpha) \exp (i \beta)|n\rangle$. Typical results, culled from numerous cases of varying $\alpha$, $\beta, k$, and $\tau$ are shown below and correspond to a weaker and stronger chaotic case, and to two values of $\beta$, i.e., $\beta=0$, and $\beta=\pi$. Specifically, we display below results for case (a) $\left|\psi_{a}^{ \pm}\right\rangle=(|+2\rangle \pm|-1\rangle) / \sqrt{2}$, with $\tau=$ $0.5, k=5.0$, and for case (b) $\left|\psi_{b}^{ \pm}\right\rangle=(|+1\rangle \pm|+2\rangle) / \sqrt{2}$, with $\tau=1.0, k=5.0$. Note that neither the basis states nor the superposition states are eigenstates of the parity operator.

Figure 1 shows $\tilde{E}$ for each of these two systems and for each of the values of $\beta$. Figure 1a, for example, displays $\tilde{E}$ for $\left|\psi_{a}^{-}\right\rangle$(dashed curve) and for $\left|\psi_{a}^{+}\right\rangle$(solid curve). Clearly, the initial state $\left|\psi_{a}^{-}\right\rangle$gives clear diffusive behavior during the first 40 kicks whereas energy absorption in the case of $\left|\psi_{a}^{+}\right\rangle$is completely suppressed. As a result, $\tilde{E}(t=40 T)=9.6$ for the $\left|\psi_{a}^{-}\right\rangle$case, while $\tilde{E}(t=40 T)=1.6$ for propagation from the initial state $\left|\psi_{a}^{+}\right\rangle$. Note (i) that this huge difference is achieved solely by changing the initial relative phase $\beta$ between the two participating states $|+2\rangle$ and $|-1\rangle$ in the initial 

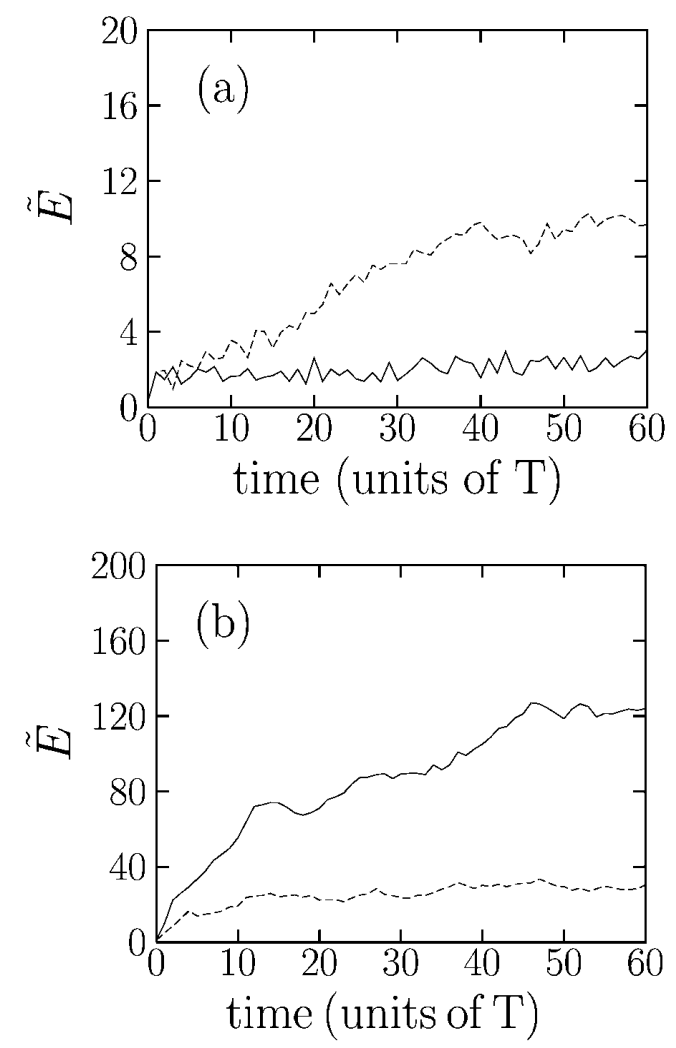

FIG. 1. The expectation value of the dimensionless scaled rotational energy $\tilde{E}=\left\langle\hat{L}^{2}\right\rangle \tau^{2} / 2 \hbar^{2}$ versus time (in units of $T$ ). (a) Solid and dashed lines are for the initial states $\left|\psi_{a}^{+}\right\rangle$and $\left|\psi_{a}^{-}\right\rangle$, respectively, $\tau=0.5, k=5.0$. (b) Solid and dashed lines are for the initial states $\left|\psi_{b}^{+}\right\rangle$and $\left|\psi_{b}^{-}\right\rangle$, respectively, $\tau=1.0$, $k=5.0$.

superposition, and (ii) that by contrast, each of $|+2\rangle$ or $|-1\rangle$ individually would behave similarly to one another with respect to energy absorption, giving $\tilde{E}(t=40 T)=$ 5.4 and 6.0, respectively. Hence, the observed control is due entirely to changing the coherent properties of the initial superposition state.

Similar control persists for the more chaotic case shown in Fig. 1b. Here $\left|\psi_{b}^{+}\right\rangle$(solid line) shows extensive chaotic diffusion (i.e., compare ordinates scale for Figs. 1a and 1b) for up to 45 kicks, giving $\tilde{E}(t=45 T)$ far higher than the value of 70.4 and 77.1 reached by propagating either of the basis functions $|+1\rangle$ and $|+2\rangle$ independently. Further, and by contrast, there is essentially no quantum diffusion after $t=4 T$ for $\left|\psi_{b}^{-}\right\rangle$(dashed line). Control (not shown) is possible for the resonant case as well, e.g., where $\tau=$ $\pi / 3$, but it is somewhat less extensive.

These differences are also reflected in the details of the evolving wave functions. For example, Fig. 2 shows the
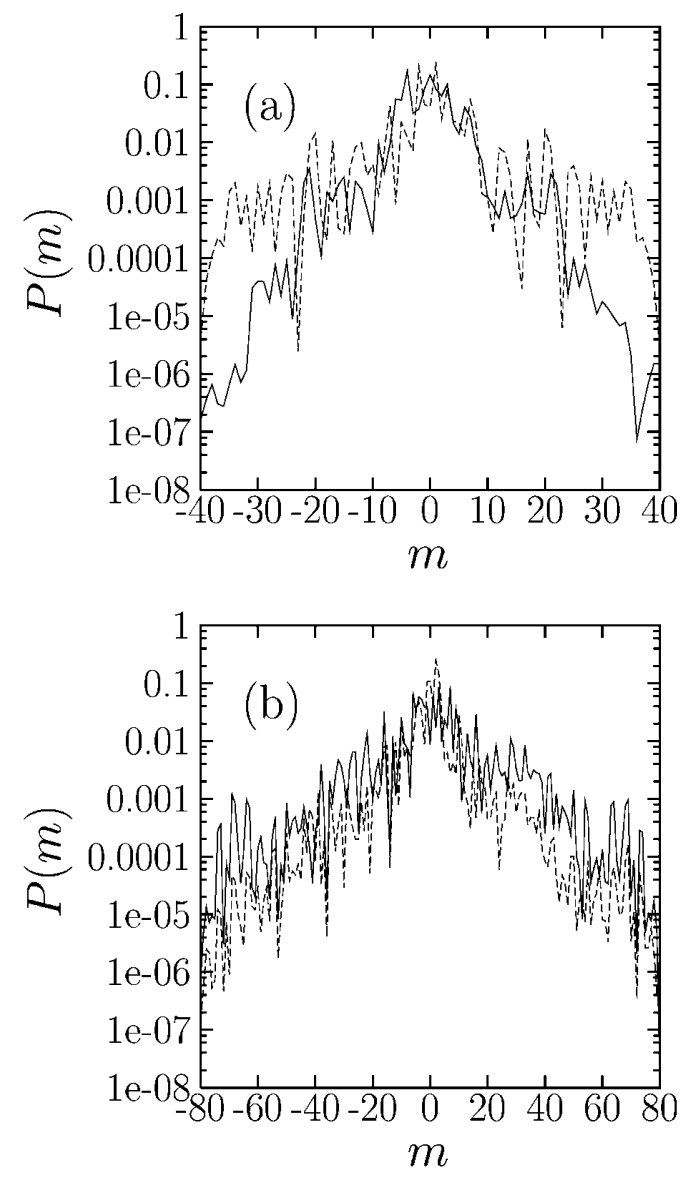

FIG. 2. Probability $P(m)$ of finding the system in the state $|m\rangle$ at $t=60 T$. Results are for the cases shown in Fig. 1 .

probability $P(m)$ of finding the system in the state $|m\rangle$ at $t=60 T$. For case (a), $P(m)$ for $|m| \geq 10$ is $15.8 \%$ and $3.4 \%$ for $\beta=\pi$ and for $\beta=0$, respectively. Similarly, for case (b) $P(m)$ differs by a factor of 5.4 for the two $\beta$ values (3.2\% vs $17.2 \%$ ) in the probability of exciting the rotor to high-energy rotational states $|m\rangle,|m| \geq 20$. In both Figs. $2 \mathrm{a}$ and $2 \mathrm{~b}$ it is evident that the difference in final populations resulting from the evolution of the two superpositions is an erratic function of $m$ with few evident trends.

The behavior shown in Fig. 1 is in sharp contrast to that which would be observed for the same initial distributions propagated classically. These computations are shown in Fig. 3 and result from classical propagation of the initially non-positive-definite Wigner function $\rho^{W}(\theta, \tilde{L})$ associated with the wave function $\cos (\alpha)|m\rangle+\sin (\alpha) \exp (i \beta)|n\rangle$. That is, we classically propagate

$$
\rho^{W}(\theta, \tilde{L})=\cos ^{2}(\alpha) \rho_{m}^{c}(\theta, \tilde{L})+\sin ^{2}(\alpha) \rho_{n}^{c}(\theta, \tilde{L})+\frac{1}{2 \pi} \sin (2 \alpha) \cos [\beta-(m-n) \theta] \delta_{\tilde{L} / \tau,(m+n) / 2},
$$

for each of $\left|\psi_{a}^{ \pm}\right\rangle$and $\left|\psi_{b}^{ \pm}\right\rangle$. In all cases, the classical results show (Fig. 3) strong diffusion, characteristic of the chaotic dynamics of the standard map. There are only small differences in the $\tilde{E}$ diffusion between $\left|\psi_{a}^{+}\right\rangle$and $\left|\psi_{a}^{-}\right\rangle$and between $\left|\psi_{b}^{+}\right\rangle$and $\left|\psi_{b}^{-}\right\rangle$. 
Consider then the origins of coherent control of chaotic systems in the quantum dynamics and the behavior in the classical limit. To this end we diagonalize the quantum map operator $\hat{F}$ by a unitary operator $\hat{U}$, i.e., $\langle i|\hat{F}| j\rangle=\sum_{k} e^{-i \phi_{k}} U_{k i}^{*} U_{k j}$, where $U_{i j} \equiv\langle i|\hat{U}| j\rangle, j=1,2, \ldots$ is the eigenvector with eigenphase $\phi_{i}$. After the initial superposition state $|\psi\rangle=$ $\cos (\alpha)|m\rangle+\sin (\alpha) \exp (i \beta)|n\rangle$ is kicked $N$ times, we have

$$
\begin{aligned}
\frac{2 \tilde{E}}{\tau^{2}}= & \cos ^{2}(\alpha) \sum_{l j j^{\prime}} l^{2} U_{j m}^{*} U_{j^{\prime} l}^{*} U_{j l} U_{j^{\prime} m} e^{i N\left(\phi_{j}-\phi_{j^{\prime}}\right)}+\sin ^{2}(\alpha) \sum_{l j j^{\prime}} l^{2} U_{j n}^{*} U_{j^{\prime} l}^{*} U_{j l} U_{j^{\prime} n} e^{i N\left(\phi_{j}-\phi_{j^{\prime}}\right)} \\
& +\left[\frac{1}{2} \sin (2 \alpha) e^{-i \beta} \sum_{l j j^{\prime}} l^{2} U_{j m}^{*} U_{j^{\prime} n} U_{j l} U_{j^{\prime} l}^{*} e^{i N\left(\phi_{j}-\phi_{j^{\prime}}\right)}+\text { c.c. }\right]
\end{aligned}
$$

where c.c. denotes the complex conjugate of the immediately preceding term within the brackets. The total term in brackets corresponds to interference effects due to initial-state coherence. For large $N$ only the $j=j^{\prime}$ terms will survive in the summations due to rapid oscillations of $e^{i N\left(\phi_{j}-\phi_{j^{\prime}}\right)}$. Hence the last two terms reduce to $1 / 2 \sin (2 \alpha) e^{-i \beta} \sum_{l} l^{2} \sum_{j}\left|U_{j l}\right|^{2} U_{j m}^{*} U_{j n}+$ c.c.

If there is no structure in $\left|U_{j l}\right|^{2}$ and the eigenvector components $U_{j m}^{*}$ and $U_{j n}$ are perfectly independent, as expected from random matrix theory [5], then $\sum_{j}\left|U_{j l}\right|^{2} U_{j m}^{*} U_{j n}$ is small and the magnitude of the interference term is $1 / \sqrt{D}$ times smaller than that of the incoherent terms (where $D$ is the effective dimension of the Hilbert space) [6], interference vanishes, and control is lost. Hence, the coherent control of quantum chaotic diffusion relies upon the residual statistical correlations between eigenvector components $\left\{U_{i j}\right\}$. Indeed, in this system the matrix $\langle i|\hat{F}| j\rangle$ is known to display a band structure
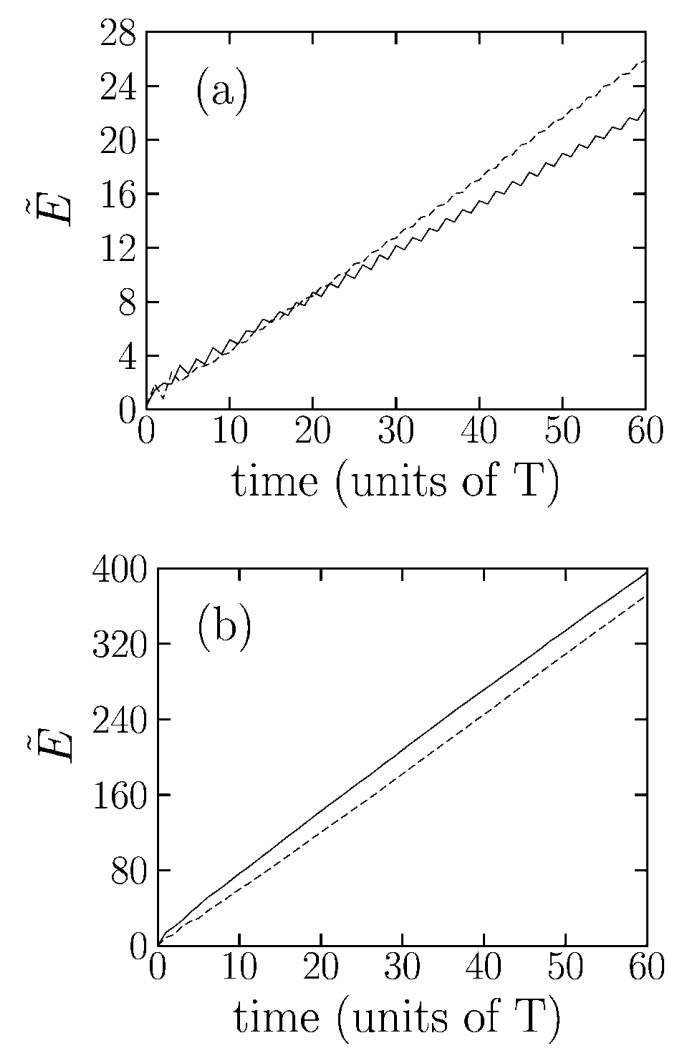

FIG. 3. As in Fig. 1 except that $\tilde{E}$ is calculated by classically propagating the initial non-positive-definite Wigner function in Eq. (4). with the bandwidth $2 k$ where the quantity $k^{2} / N$, where $N$ is the size of the banded random matrices, provides a measure of the statistical deviations from random matrix theory [7]. Numerical results show that $k^{2} / N<0.2$ is sufficiently small for control to persist. This being the case, we obtain a necessary condition to ensure the significance of the interference term, namely, $k<0.2 N_{\kappa} / \kappa$ or $\tau>\kappa^{2} /\left(0.2 N_{\kappa}\right)$, where $N_{\kappa}$ is the minimum grid size for accurate fast-Fourier transform calculations with $\tau=1$. Numerical studies indicate that, for $\kappa<10.0, N_{\kappa} \approx 256$, implying that we require $k<50 / \kappa$ or $\tau>\kappa^{2} / 50$ for control. This makes it clear that as one approaches the classical limit (by increasing $k$ or decreasing $\tau$ with fixed $\kappa$ ), coherent control is lost.

Further evidence that deviations from random matrix theory are responsible for control was obtained by examining control using a model composed of a banded matrix with random matrix elements. Control was obtained in this case as well, but was not as extensive as the kicked rotor system since the latter displays less random matrix character.

The dynamics of the kicked rotor in the presence of decoherence effects has also been examined both experimentally [8] and theoretically [9]. The survival of control in the presence of decoherence is of interest both in general and for this particular case. To examine this issue we introduce a simple decoherence model. Here, the quantum map operator between $(N-1 / 2) T$ and $(N+1 / 2) T$ is taken as $\hat{R} \hat{F}$, where $\hat{R}$ introduces random phases into the system. Specifically, $\hat{R}|m\rangle=e^{i 2 \pi r \xi(m, N)}|m\rangle$, where $m=0, \pm 1, \pm 2, \ldots$, and $\xi(m, N)$ takes on random values that are distributed uniformly between 0 and 1 for each different $m$ or $N$. Note that this model is such that its $r \rightarrow 1$ limit corresponds to measurement-induced quantum diffusion [10].

The density matrix $\hat{\rho}$ for the dynamics governed by $\hat{R} \hat{F}$ can then be obtained as an average over many realizations of $\xi(m, N)$. We take the linear entropy $S \equiv \operatorname{Tr} \hat{\rho}^{2}$ as a useful additional measure of the purity of quantum states and hence of the effect of decoherence.

Numerical studies show that for $r<0.05$, coherent control of quantum diffusion is hardly affected by the decoherence. For stronger decoherence, e.g., $r=0.15$, phase control is essentially lost. Examination of the corresponding values of $S(t=60 T)$ shows that this is consistent with maintenance of control when the decoherence is sufficiently small so that $S(t=60 T)>0.4$. Sample results 

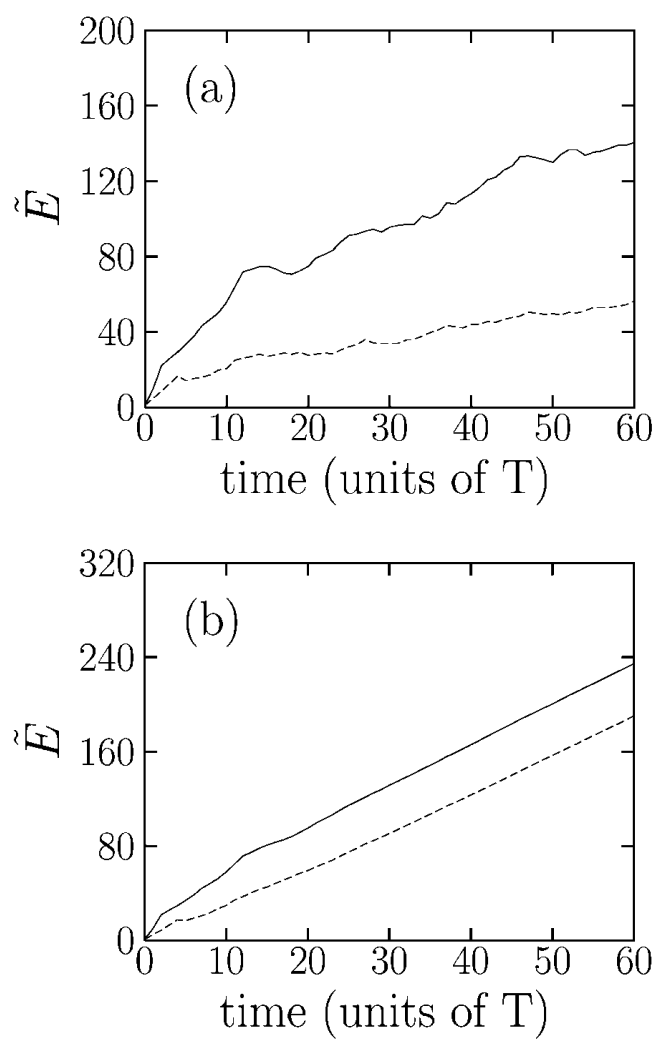

FIG. 4. As in Fig. 1b but in the presence of (a) modest decoherence and (b) stronger decoherence.

are shown in Fig. 4 where we plot the time dependence $\tilde{E}$ versus the time in the presence of small decoherence (Fig. 4a, $\quad r=0.05$ ) and of appreciable decoherence (Fig. 4b, $r=0.15$ ). Both panels refer to the case of stronger chaos. Comparison of Fig. $4 \mathrm{a}$ with the decoherence-free dynamics in Fig. $1 \mathrm{~b}$ shows that control is still significant, but decoherence is beginning to have an effect insofar as $\tilde{E}$ at $t=60 T$ is larger in Fig. 4a than in Fig. 1b. That is, the results show a slight tendency towards the classical behavior. In the case of stronger decoherence (Fig. 4b) phase control is greatly reduced and long time linear diffusive growth of $\tilde{E}$ is observed. A careful examination of Fig. 4b suggests that phase control persists until $t \approx 20 T$ when the slopes of the dashed and solid curves become virtually identical. However, the slopes of these curves are still significantly less than those in Fig. 3b, suggesting that quantum coherence is still maintained at these longer times. In essence, it appears that phase control over the diffusion rate vanishes before quantum coherence is completely destroyed.

Note, finally, that the possibility of control does not rely heavily on the specific choice of basis states [11]. For example, we have also obtained extensive phase control by adding together either negative parity real basis states $\sin (m \theta) / \sqrt{\pi}$ or by adding together positive parity states $\cos (m \theta) / \sqrt{\pi}$. Since the states $\sin (m \theta) / \sqrt{\pi}$ satisfy the boundary condition of an infinitely deep square well potential $V(\theta)$ with $V(0)=V(2 \pi)=+\infty$ and since the dy- namics of a kicked particle in a well is similar to the kicked rotor for similar initial states [12], this indicates that one can also demonstrate coherent control of chaotic diffusion using superpositions of Hamiltonian eigenstates of a kicked particle in a potential well.

A number of possible experimental demonstrations of the proposed control scenario are evident. For example, the kicked diatomic molecule $C s I[6,13]$ is a promising molecular system for demonstrating controlled quantum chaotic diffusion. In this case, preliminary controlled laser excitation could be used to prepare the desired initial superposition state (which are here superpositions of $|J, M\rangle$ and $\left|J^{\prime}, M\right\rangle$, where $J$ and $J^{\prime}$ are the angular momentum and $M$ is their projection on the $z$ axis) and to vary $\beta$. Alternatively, one can utilize the square well analogy described above to experimentally study kicked dynamics of a particle in a well. By contrast, implementation of the atom-optics approach [14] to studies of control appears more difficult, insofar as it is necessary to prepare initial quantum superposition states, a considerable extension of previous work [15].

This work was supported by the U.S. Office of Naval Research and the Natural Sciences and Engineering Research Council of Canada. We thank Professor Aephraim Steinberg for discussions on the atom-optics approach to $\delta$ kicked dynamics.

[1] G. Casati and B. Chirikov, Quantum Chaos: Between Order and Disorder (Cambridge University Press, New York, 1995).

[2] M. Shapiro and P. Brumer, Adv. At. Mol. Opt. Phys. 42, 287 (2000); P. Brumer and M. Shapiro, Sci. Am. 272, No. 3, 34 (1995).

[3] D. L. Shepelyansky, Physica (Amsterdam) 28D, 103 (1987).

[4] For example, C. Jaffe and P. Brumer, J. Chem. Phys. 82, 2330 (1985).

[5] F. Haake, Quantum Signatures of Chaos (Springer-Verlag, Berlin, 1992).

[6] J. Gong and P. Brumer (to be published).

[7] G. Casati, I. Guarneri, F. M. Izrailev, and R. Scharf, Phys. Rev. Lett. 64, 5 (1990).

[8] B. G. Klappauf et al., Phys. Rev. Lett. 81, 1203 (1998); H. Ammann et al., Phys. Rev. Lett. 80, 4111 (1998).

[9] E. Ott, T. M. Antonsen, Jr., and J. D. Hanson, Phys. Rev. Lett. 53, 2187 (1984).

[10] P. Facchi, S. Pascazio, and A. Scardicchio, Phys. Rev. Lett. 83, 61 (1999).

[11] We have also obtained similar control results with $\cos \theta$ in Eq. (1) replaced by $\cos (\theta+\pi / 10)$, which gives a Hamiltonian that does not conserve parity.

[12] R. Sankaranarayanan, A. Lakshminarayan, and V.B. Sheorey, preprint nlin 0005035.

[13] R. Blümel, S. Fishman, and U. Smilansky, J. Chem. Phys. 84, 2604 (1986).

[14] R. Graham, M. Schlautmann, and P. Zoller, Phys. Rev. A 45, R19 (1992).

[15] F. L. Moore et al., Phys. Rev. Lett. 75, 4598 (1995). 